\title{
Conexões entre as temáticas Objetivos de Desenvolvimento Sustentável (ODS), Indicadores de Sustentabilidade e Gestão Municipal Sustentável: Uma revisão sistemática da literatura contemporânea
}

\author{
Connections between theme Development Objectives Sustainable (SDG), Sustainability Indicators \\ and Sustainable Municipal Management: A systematic review of contemporary literature
}

Conexiones entre los temas Objetivos de Desarrollo Sostenible (ODS), Indicadores de

Sostenibilidad y Gestión Municipal Sostenible: Una revisión sistemática de la literatura

contemporânea

Recebido: 21/04/2021 | Revisado: 03/05/2021 | Aceito: 04/05/2021 | Publicado: 17/05/2021

\begin{abstract}
Anna Karolina Marinho de Menêzes ORCID: https://orcid.org/0000-0002-2964-0912 Universidade Federal de Campina Grande, Brasil E-mail: anna.karolina.menezes@gmail.com

Maria de Fátima Martins ORCID: https://orcid.org/0000-0002-9578-9555 Universidade Federal de Campina Grande, Brasil E-mail: fatimamartins2005@gmail.com
\end{abstract}

\begin{abstract}
Resumo
O estudo sobre Desenvolvimento Sustentável é um campo científico que vem sendo desenvolvido e trabalhado em diversos aspectos, caracterizado como multidisciplinar e multifacetado. Os indicadores de sustentabilidade são ferramentas essenciais na tentativa de mensurá-lo, impulsionados pelo desejo de se alcançar os ODS's, sendo as cidades, a gestão e a população potenciais aliados para a sua promoção. Nesse sentido, compreender e discutir a complexidade que envolve tal temática é condição para que se consiga avançar efetivamente nesse campo de pesquisa de forma aprofundada. Com base nisso, este artigo teve como objetivo analisar a produção científica recente sobre os Objetivos de Desenvolvimento Sustentável, os Indicadores de Sustentabilidade e a Gestão Municipal Sustentável, a fim de compreendê-los e identificar os principais contextos que orbitam sobre os temas em estudo. Para tanto, fez-se uso da plataforma Web of Science, para a coleta de dados, adotando, como procedimento de pesquisa, a revisão sistemática da literatura e, como ferramentas de análise, fez-se uso do software CitNetExplorer. Os resultados permitiram categorizar os trabalhados explorados em 7 clusters, agrupados de acordo com a sua temática central, viabilizando, assim, o alcance do objetivo proposto. A base de dados utilizada também revelou temáticas transversais às propostas nesse estudo, que poderão, de forma significativa, auxiliar no desenvolvimento de outras pesquisas.
\end{abstract}

Palavras-chave: Desenvolvimento sustentável; Indicadores de sustentabilidade; Gestão municipal sustentável.

\begin{abstract}
The study on Sustainable Development is a scientific field that has been developed and worked on in several aspects, characterized as multidisciplinary and multifaceted. Sustainability indicators are essential tools in an attempt to measure it, driven by the desire to reach the SDGs, with cities, management and the population being potential allies for their promotion. In this sense, understanding and discussing the complexity that surrounds this theme is a condition for effectively advancing this field of research in depth. Based on this, this article aimed to analyze the recent scientific production on the Sustainable Development Goals, Sustainability Indicators and Sustainable Municipal Management, in order to understand them and identify the main contexts that orbit about the topics under study. For this, the Web of Science platform was used for data collection, adopting the systematic literature review as a research procedure and as analysis tools, the CitNetExplorer software was used. The results allowed to categorize the works explored in 7 clusters, grouped according to their central theme, thus enabling the achievement of the proposed objective. The database used also revealed themes transversal to the proposals in this study, which could significantly assist in the development of other research.
\end{abstract}

Keywords: Sustainable development; Sustainability indicators; Sustainable municipal management. 


\begin{abstract}
Resumen
El estudio sobre Desarrollo Sostenible es un campo científico que ha sido desarrollado y trabajado en varios aspectos, caracterizados como multidisciplinares y multifacético. Los indicadores de sostenibilidad son herramientas esenciales en un intento de medirlo, impulsado por el deseo de alcanzar los ODS, con ciudades, gestión y potenciales aliados para su promoción. En este sentido, comprender y discutir el La complejidad que involucra tal tema es una condición para avanzar de manera efectiva en este campo de investigación en profundidad. Con base en esto, este artículo tenía como objetivo analizar la producción científica reciente sobre los Objetivos de Desarrollo Sostenible, la Indicadores de Sostenibilidad y Gestión Municipal Sostenible, con el fin de comprenderlos y Identificar los principales contextos que orbitan sobre los temas en estudio. Para eso se usó de la plataforma Web of Science, para la recolección de datos, adoptando, como procedimiento de investigación, la revisión sistemática de la literatura y, como herramientas de análisis, se utilizó la Software CitNetExplorer. Los resultados permitieron categorizar a los trabajadores explorados en 7 agrupaciones, agrupadas de acuerdo con su tema central, permitiendo así el alcance de la objetivo propuesto. La base de datos utilizada también reveló temas transversales a la propuesto en este estudio, que podría ayudar significativamente en el desarrollo de otras investigaciones.
\end{abstract}

Palabras clave: Desarrollo sostenible; Indicadores de sostenibilidad; Gestión municipio sostenible.

\title{
1. Introdução
}

As discussões sobre sustentabilidade vêm ganhando mais evidência diante da urgência em conciliar desenvolvimento e sustentabilidade. Jacobi (1999, p. 175) acrescenta ao dizer que "o quadro socioambiental que caracteriza as sociedades contemporâneas revela que o impacto dos humanos sobre o meio ambiente estão se tornando cada vez mais complexos, tanto em termos quantitativos quanto qualitativos".

Apesar dos reconhecidos avanços no âmbito do debate sobre desenvolvimento e sustentabilidade, ainda impera o dilema da adequação política entre fins e meios (Réus \& Andion, 2018). Isto é, prevalece a discrepância entre os princípios e as formas de gestão e estratégias de ação, em outras palavras, prepondera o distanciamento entre a teoria e a práxis.

Diante da complexidade e intangibilidade que permeiam a sustentabilidade, Marques, Santos e Aragão (2020) entendem que é imperativo que haja uma intervenção, no sentido de adequar todas as dimensões que envolvem a sustentabilidade para que de fato o desenvolvimento siga para a direção que se pretende.

Mas, para que isso se torne possível, é necessário enxergar o desenvolvimento sustentável sob várias óticas, "contemplando os aspectos econômicos, políticos, ecológicos, sociológicos, espaciais e culturais, através da interrelação de indivíduos e múltiplas áreas do conhecimento" (Marques et al., 2020, p. 17).

Em suma, pode-se dizer que a relação entre os empasses globais de sustentabilidade e as ações locais compõem o desafio central da atualidade. Sendo assim, este estudo explora essa problemática, analisando as pesquisas mais recentes que abordam três temas relevantes nessa discussão - Objetivos de Desenvolvimento Sustentável, Indicadores de Sustentabilidade e Gestão Municipal Sustentável.

Buscando identificar o caminho traçado pelos pesquisadores desses temas, adotou-se a metodologia de revisão sistemática de literatura para mapear os artigos mais relevantes, de forma a responder a seguinte questão de pesquisa: " $E m$ quais contextos estão sendo trabalhados, nas pesquisas científicas contemporâneas, os objetivos de Desenvolvimento Sustentável, os Indicadores de Sustentabilidade e a Gestão Municipal Sustentável?”.

Sendo assim, para responder a essa questão, este artigo tem como objetivo principal analisar a produção científica recente sobre os temas - Objetivos de Desenvolvimento Sustentável, Indicadores de Sustentabilidade e Gestão Municipal Sustentável - a fim de compreendê-la e identificar os principais contextos que orbitam sobre os temas em estudo.

A relevância desta pesquisa se dá por mapear os estudos atuais e de maior contribuição científica relacionados aos temas propostos, possibilitando a outros pesquisadores um panorama atual da área de estudo. Para tanto, este artigo estruturase em cinco partes: os aspectos introdutórios, uma breve conceituação dos temas estudados, os procedimentos metodológicos utilizados para concretizar o estudo, os resultados encontrados e as considerações finais. 


\section{Procedimentos Metodológicos}

O presente estudo objetivou realizar um mapeamento da produção científica atual, sobre Objetivos de Desenvolvimento Sustentável, Indicadores de Sustentabilidade e Gestão Municipal Sustentável, a partir de uma revisão sistemática de literatura (RSL) que, de acordo com Kitchenham e Charles (2007), é uma modalidade de pesquisa que usa como fonte de dados a literatura sobre determinado tema, e é especialmente útil para integrar as informações sobre determinado assunto, as quais podem apresentar resultados conflitantes e/ou coincidentes, como também pontuar temas para investigações futuras.

Inicialmente, foi gerado um banco de dados no dia 14 de agosto de 2020, na plataforma Web of Science (WOS), onde sua escolha se deu tanto pela diversidade e qualidade do periódico, o que garante uma maior relevância dos artigos, como pelo fato de que o software CitNetExplorer - escolhido para selecionar os objetos de estudo - restringe-se a artigos provenientes dessa base.

O procedimento de busca se deu através da pesquisa pelos seguintes termos relacionados ao tema em estudo: "sustainable municipal management", "municipal sustainability indicators", "sustainability indicators for municipal management", "municipal management and sustainable development", "municipal sustainable development", "sustainable development goals in city", "sustainable city management" e "sustainable development goals".

Considerando todos os termos citados e o intervalo dos anos de 1975 a 2020, a pesquisa inicial retornou 7.500 documentos. Para refinamento da base dos dados, foram filtrados artigos em inglês, espanhol e português pertencentes às categorias "administração pública", "estudos ambientais", "estudos urbanos" e "planejamento urbano regional", nos anos de 2017 a 2020, tendo em vista que, são os anos com maior número de publicações na área em estudo (>100/ano), como mostra a Figura 3. Desse modo, o número final de artigos utilizados no escopo do estudo foi de 952.

Figura 1 - Distribuição das publicações 2011-2020.

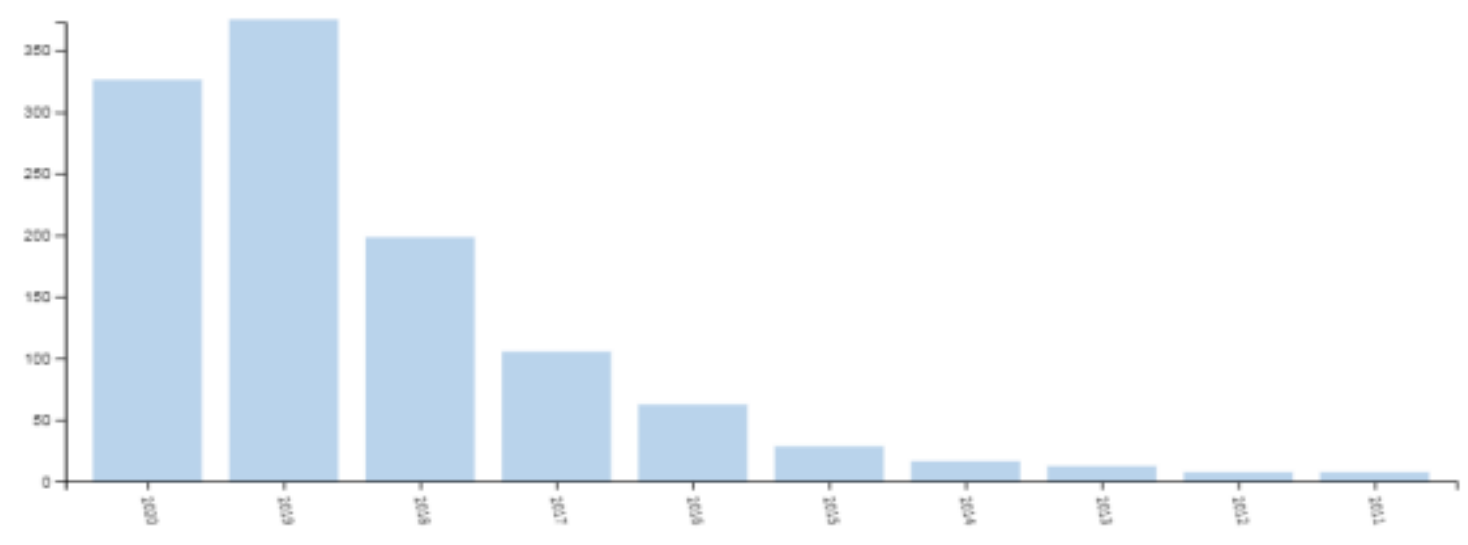

Fonte: Autores, adaptado de Web of Science (2020).

Visando o atingir o objetivo proposto, foi realizada uma análise bibliométrica a partir do software CitNetExplorer, que permitiu visualizar e selecionar os objetos de estudo. Inicialmente, o software gerou uma rede (Figura 4) com 952 publicações e 474 links de citação no recorte temporal de 4 anos (2107-2020), excluindo as publicações que estão como referências citadas nas publicações da busca. Nessa rede inicial, o número de publicações visualizadas foi alterado para 100. 
Figura 2 - Rede inicial.

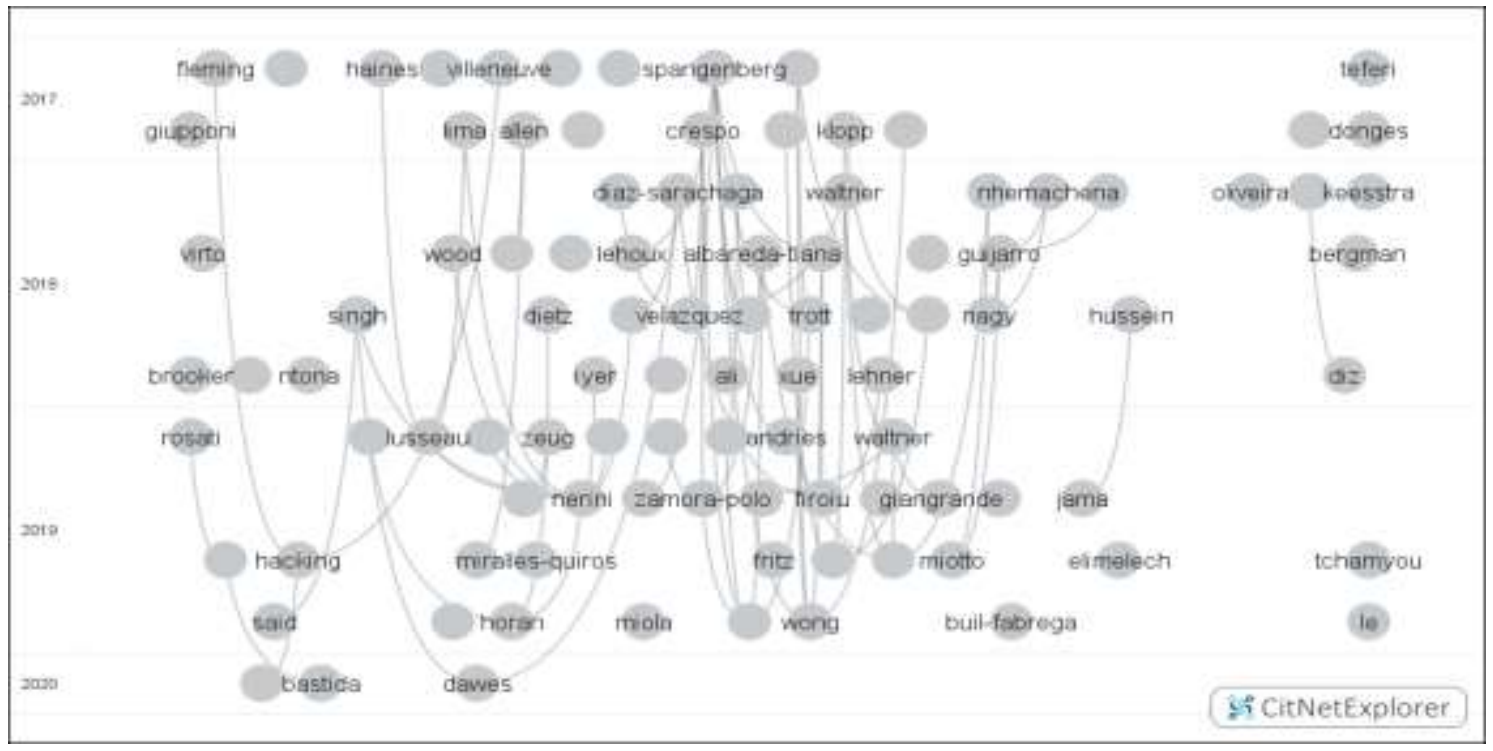

Fonte: Autores, adaptado de CitNetExplorer (2020).

Após essa primeira visualização, foram selecionadas as publicações mais relevantes por meio da análise Core Publications, que retornou um quantitativo de 393 publicações com pelo menos um link de citação com outro core publications. Em seguida, foi feita uma análise de Clustering no Core Publications, mantendo os parâmetros padrão do software, a análise (Figura 5) informa que 7 clusters foram formados e que 665 publicações não pertencem a nenhum cluster.

Figura 3 - Análise de Clustering.

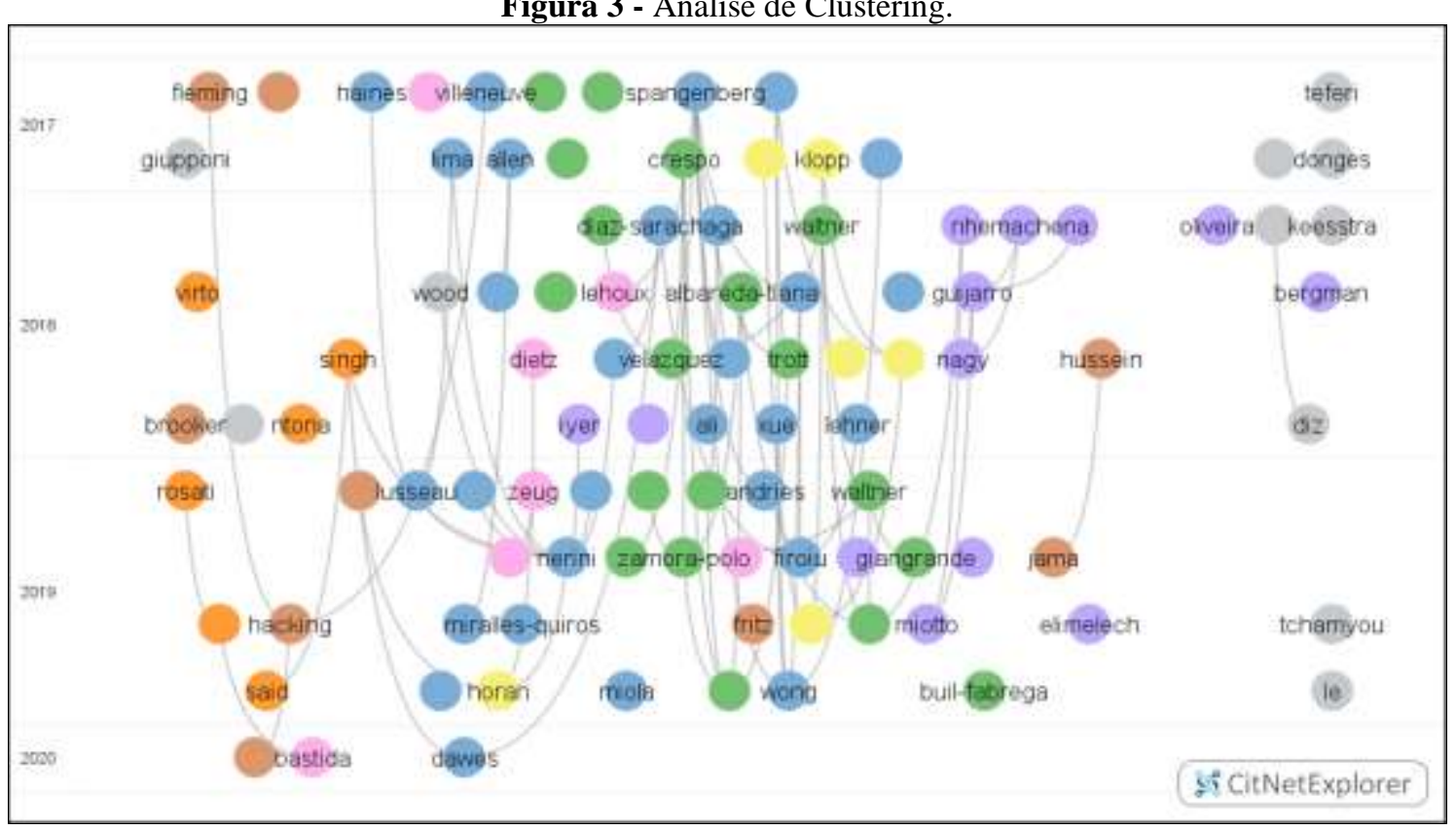

Fonte: Autores, adaptado de CitNetExplorer (2020).

A análise da rede obtida no CitNetExplorer foi feita a partir dos 7 clusters formados. Para analisá-los, foram elaboradas 7 tabelas (uma para cada cluster), contendo as 3 principais publicações de cada grupo, ou seja, as 3 publicações com maior score de cada cluster. Para a construção dessas tabelas, foram realizadas as análises dos 21 abstracts referentes as 21 publicações selecionadas, a partir do grau de relevância em cada cluster. 
Por fim, com o propósito de facilitar a compreensão acerca de Desenvolvimento Sustentável - ainda conflitante na literatura - foram realizadas análises mais aprofundadas (não apenas dos abstracts) dos principais artigos da base de dados escolhida, obtidos partir do h-index no próprio software, o qual caracteriza-se como uma proposta para quantificar a produtividade e o impacto dos investigadores, baseando-se nos seus artigos mais citados (Lopes, Costa, Fernández-Llimós, Amante, \& Lopes, 2012, p. 3).

\section{Objetivos de Desenvolvimento Sustentável}

O termo Desenvolvimento Sustentável foi discutido pela primeira vez em 1983, por ocasião da Comissão Mundial sobre Meio Ambiente e Desenvolvimento, criada pela ONU, onde foi proposto que o desenvolvimento econômico fosse integrado à questão ambiental. A partir de então, presenciou-se um crescimento significativo no interesse da sociedade em relação ao meio ambiente decorrente da difusão do termo "desenvolvimento sustentável", que, segundo Van Bellen (2004), surgiu mediante o aprofundamento da crise ambiental juntamente com a reflexão sistemática sobre a influência da sociedade neste processo.

Ainda de acordo com o autor supracitado, esse novo conceito alcançou um destaque inusitado a partir da década de 1990, tornando-se um dos termos mais utilizados para se definir um novo modelo de desenvolvimento. Carvalho e Barcellos (2010, p. 101) traz o conceito de desenvolvimento sustentável mais usual, definido como sendo "aquele que atende às necessidades do presente sem comprometer a possibilidade de gerações futuras atenderem a suas próprias necessidades". Ou seja, consiste em usar os recursos naturais com respeito e consciência, sendo caracterizado como um desenvolvimento que não esgota os recursos, mas visa à conciliação entre crescimento econômico e preservação da natureza.

Na tentativa de operacionalizar o Desenvolvimento Sustentável, os Objetivos de Desenvolvimento Sustentável (ODS), propostos pela Organização das Nações Unidas (ONU), se destacam. De acordo com a Organização das Nações Unidas ( $s . d$.), os ODS se concretizaram como a maior iniciativa planetária, voltada à redução da pobreza e das desigualdades, em busca de melhoria nas condições sociais e econômicas, unindo a promoção dos direitos humanos, a proteção do meio ambiente e do clima.

Para Menezes e Minillo (2017), os ODS concebem metas importantes, que permitem criar uma agenda ampla e eficaz, na pretensão de alcançar um patamar revolucionário. Mas, para que essa agenda ocorra de maneira eficiente e obtenha êxito, é imprescindível, ao processo, alinhar os ODS à realidade local e incluir a participação ativa da população. Ainda segundo os autores, três elementos são especialmente importantes: a conformação local de discursos globais, o controle e fiscalização das ações governamentais e o cumprimento das metas por parte da população.

\section{Indicadores de Sustentabilidade}

O termo indicador é originário do latim indicare, que significa descobrir, apontar, anunciar, estimar, de acordo com a definição de Hammond (1995). No que tange à sustentabilidade, esses indicadores são ferramentas utilizadas no auxílio ao desenvolvimento, sendo a principal contribuição disponibilizar informações sobre o estado das diversas dimensões (ambientais, econômicas, socioeconômicas, culturais, institucionais, etc.) que compõem o desenvolvimento sustentável (Carvalho, Curi, Carvalho \& Curi, 2011).

De acordo com Van Bellen (2006), a principal função dos indicadores é reunir e quantificar informações, simplificando as mais complexas e tentando melhorar, desse modo, a comunicação. Sendo assim, uma das grandes aplicações dos indicadores consiste no monitoramento do progresso nas distintas dimensões que compõe a sustentabilidade, pois funcionam como ferramenta de apoio aos tomadores de decisões e na elaboração de políticas de fomento à sustentabilidade (Kemerich, Ritter \& Borba, 2014). 
Os indicadores ainda permitem avaliar condições e tendências, comparar lugares e situações, prover informações de advertência e antecipar condições e tendências (Van Bellen, 2006). Para Gallopin (1996), os indicadores mais adequados são aqueles que resumam ou simplifiquem as informações mais relevantes, fazendo com que os fenômenos reais se tornem mais visíveis. Em outras palavras, os indicadores precisam agregar e quantificar informações de modo que sejam significativos, viáveis e traduzam a realidade.

É sabido que o conceito de sustentabilidade é marcado por polêmicas e controvérsias, tendo em vista esse conflito, os indicadores de sustentabilidade vêm para contribuir na tentativa de mensurar e dar mais clareza ao conceito. A criação de um instrumento de medição da sustentabilidade, capaz de apresentar o grau de sustentabilidade de uma população, monitorar tendências e auxiliar no estabelecimento de metas, sempre foi um desafio no alcance do desenvolvimento sustentável. Assim, "os indicadores são, portanto, instrumentos essenciais para guiar a ação e subsidiar o acompanhamento e a avaliação do progresso alcançado rumo à sustentabilidade" (Polaz \& Teixeira, 2009, p. 3).

\section{Gestão Municipal Sustentável}

No final do século passado, o pesquisador Ignacy Sachs passou a denunciar os limites do desenvolvimento como advindo apenas do crescimento econômico e a incluir outros fatores - ambiental, social, política, cultural - nas discussões. Essas dimensões passaram a ocupar papel essencial na composição do planejamento governamental e das políticas públicas no mundo, tendo como fim a promoção da sustentabilidade (Sachs, 1980, 1986, 1997, 2000 como citado em Réus \& Andion, 2018).

No que tange à criação de uma Gestão Pública voltada para Sustentabilidade, Schenini e Nascimento $(2002$, p. 10) são enfáticos ao dizer que: "a premissa básica é a conciliação dos conflitos entre proteção ambiental, desenvolvimento econômico e justiça social, através de um processo contínuo". Tornando-se, assim, imprescindível uma nova abordagem do planejamento público, pautada em um processo constante e participativo, que propicie identificar os problemas da localidade, e promova o uso correto dos recursos naturais, a otimização dos serviços públicos e o bem-estar da população. Em outras palavras, um processo que busque:

Orientar o crescimento físico e socioeconômico das cidades, bem como de sua expansão, com o estímulo das principais funções e atividades urbanas como habitação, trabalho, transportes, educação, saúde, lazer, indústria, comércio e serviços, associados à preservação, proteção e recuperação dos valores históricos, culturais, paisagísticos e ambientais (Fórum AG21, 2000).

Há várias formas de conduzir a gestão pública ecologicamente correta, sendo que os principais papéis são "o de fiscalizador dos destruidores e o de fomentador das atividades que tragam menor destruição, mudanças culturais ou aproveitamento dos recursos naturais" (Schenini \& Nascimento, 2002, p. 17). Por fim, Moura (1998) ratifica ao inferir que o município se torna um campo privilegiado para a promoção do desenvolvimento sustentável, pela proximidade que a esfera local tem com os cidadãos e suas necessidades.

\section{Resultados e Discussões}

Nessa etapa realizou-se as análises das publicações mais importantes - de acordo com o score disponibilizado no software CitNetExplorer - ou seja, aquelas que estão contribuindo de maneira significativa no campo da ciência. Ademais, a interface do software auxiliou na identificação das temáticas dos clusters gerados. 


\subsection{Dos clusters}

O primeiro cluster, representado pela cor azul na Figura 3, é o grupo com maior número de publicações (87), nele também está a publicação com maior score (15), como mostra a Tabela 1. Diante da análise dos abstracts dos artigos mais importantes desse cluster, pode-se perceber que essa área de estudo foca em avaliar com maior criticidade os Objetivos de Desenvolvimento Sustentável (ODS), portanto, o cluster foi denominado de: Um Olhar Crítico sob os ODS.

Tabela 1 - Cluster 1 (Azul),

\begin{tabular}{|c|c|c|c|}
\hline $\begin{array}{c}\mathrm{N}^{\circ} \text { de } \\
\text { Publicações }\end{array}$ & $\begin{array}{c}\text { Autores com maior } \\
\text { score }\end{array}$ & Artigo & Análise do Abstract \\
\hline \multirow{3}{*}{$\begin{array}{l}1^{\circ} \text { Cluster: } \\
87\end{array}$} & $\begin{array}{l}\text { Joachim H. Spangenberg } \\
\text { (score 15) }\end{array}$ & $\begin{array}{l}\text { Hot Air or Comprehensive } \\
\text { Progress? A Critical } \\
\text { Assessment of the SDGs. }\end{array}$ & $\begin{array}{l}\text { Analisa os ODS quanto à sua } \\
\text { coerência e seus impactos na sua } \\
\text { implementação. }\end{array}$ \\
\hline & $\begin{array}{l}\text { Erling Holden; Kristin } \\
\text { Linnerud; } \\
\text { David Banister (score 11) }\end{array}$ & $\begin{array}{l}\text { The Imperatives of Sustainable } \\
\text { Development. }\end{array}$ & $\begin{array}{l}\text { Sugere um novo modelo para o } \\
\text { desenvolvimento sustentável. }\end{array}$ \\
\hline & $\begin{array}{l}\text { Jose Manuel Diaz- } \\
\text { Sarachaga; Daniel Jato- } \\
\text { Espino; Daniel Castro- } \\
\text { Fresno (score 11) }\end{array}$ & $\begin{array}{l}\text { Is the Sustainable Development } \\
\text { Goals (SDG) index an adequate } \\
\text { framework to measure the } \\
\text { progress of the } 2030 \text { Agenda? }\end{array}$ & $\begin{array}{l}\text { Busca analisar a adequação do "SDG } \\
\text { Index" para avaliar o cumprimento da } \\
\text { Agenda } 2030 \text {. }\end{array}$ \\
\hline
\end{tabular}

Fonte: Autores, adaptado de CitNetExplorer (2020).

O cluster, representado pela cor verde na Figura 3, é o segundo grupo com maior número de publicações (51). Na Tabela 2, estão as publicações mais relevantes desse cluster, que após a análise dos seus abstracts, foi possível entender que o assunto predominante nessa linha de pesquisa é a educação voltada para a sustentabilidade, sendo assim, denominou-se o grupo de: Educação para a Sustentabilidade.

Tabela 2 - Cluster 2 (Verde).

\begin{tabular}{|c|c|c|c|}
\hline $\begin{array}{c}\mathrm{N}^{\bullet} \text { de } \\
\text { Publicaçōes }\end{array}$ & $\begin{array}{l}\text { Autores com maior } \\
\text { score }\end{array}$ & Artigo & Análise do Abstract \\
\hline \multirow{3}{*}{$2^{\circ}$ Cluster: } & $\begin{array}{l}\text { Eva-Maria Waltner; } \\
\text { Werner Riess; Christoph } \\
\text { Mischo (score 6) }\end{array}$ & $\begin{array}{l}\text { Development and Validation of } \\
\text { an Instrument for Measuring } \\
\text { Student Sustainability } \\
\text { Competencies. }\end{array}$ & $\begin{array}{l}\text { Desenvolve um método para } \\
\text { avaliaçāo de competencias/produção } \\
\text { de sustentabilidade entre alunos. }\end{array}$ \\
\hline & $\begin{array}{l}\text { Bärbara Crespo; Carla } \\
\text { Miguez-Alvarez; Maria } \\
\text { Elena Arce; Miguel } \\
\text { Cuevas; José Luis } \\
\text { Miguez (score 5) }\end{array}$ & $\begin{array}{l}\text { The Sustainable Development } \\
\text { Goals: An Experience on } \\
\text { Higher Education. }\end{array}$ & $\begin{array}{l}\text { Analisa a capacidade dos alunos de } \\
\text { incorporar os prineipios da } \\
\text { sustentabilidade em seus trabalhos. }\end{array}$ \\
\hline & $\begin{array}{l}\text { Silvia Albareda-Tiana; } \\
\text { Salvador Vidal-Raméntol; } \\
\text { Maria Pujol-Valls; } \\
\text { Mónica Fernaindez- } \\
\text { Morilla (score 5) }\end{array}$ & $\begin{array}{l}\text { Holistic Approaches to Develop } \\
\text { Sustainability and Research } \\
\text { Competencies in Pre-Service } \\
\text { Teacher Training. }\end{array}$ & $\begin{array}{l}\text { Explora as metodologias de ensino } \\
\text { para o desenvolvimento de } \\
\text { competéncias em sustentabilidade no } \\
\text { ensino superior. }\end{array}$ \\
\hline
\end{tabular}

Fonte: Autores, adaptado de CitNetExplorer (2020).

A cor lilás na Figura 3, representa o terceiro cluster com mais publicações (46), como evidenciado na Tabela 3. Diante da investigação feita nos abstracts desse grupo, tornou-se compreensível que a área explorada se volta para os aspetos socioeconômicos da sustentabilidade, ressaltando os indicadores de sustentabilidade como um mecanismo medidor. Assim, esse cluster foi nominado como: Mensurando o Desenvolvimento Sustentável à luz de Indicadores de Sustentabilidade. 
Tabela 3 - Cluster 3 (Lilás).

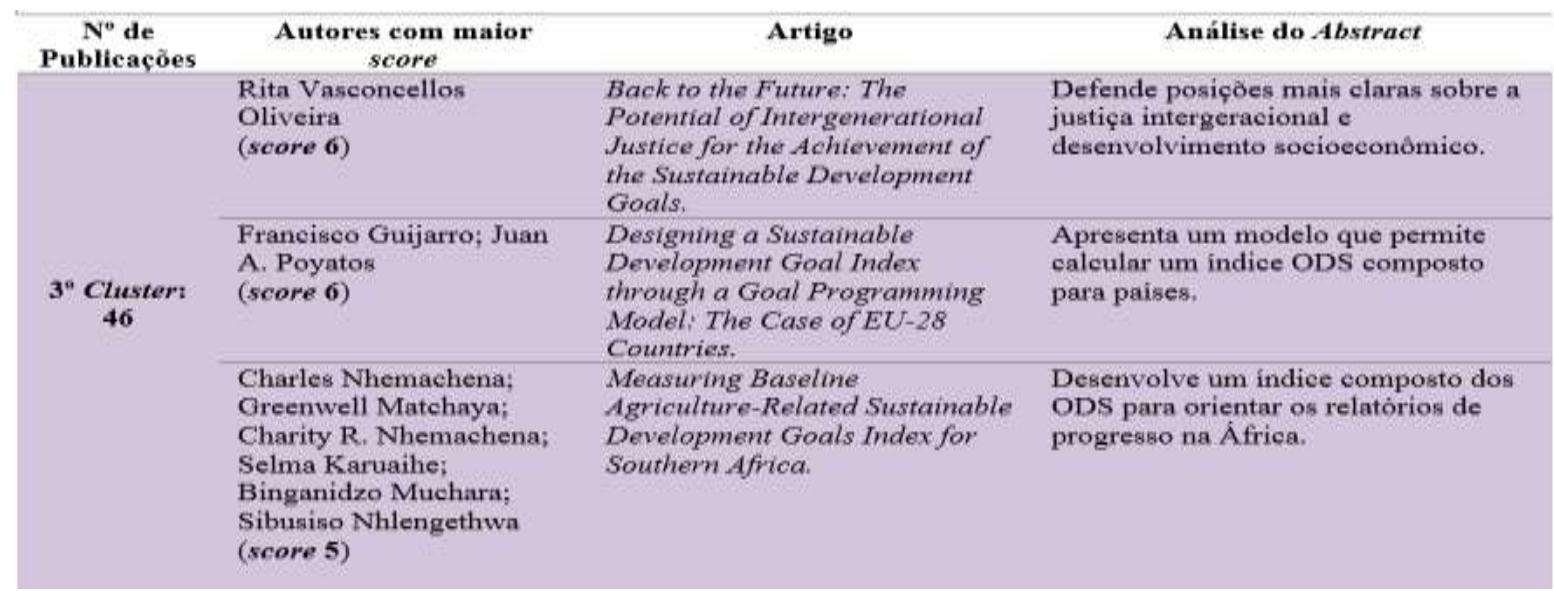

Fonte: Autores, adaptado de CitNetExplorer (2020).

O quarto cluster é representado pela cor laranja na Figura 3, nele estão 37 publicações com scores que variam de 4 a 15, como mostra a Tabela 4. Diante da análise dos abstracts dos três artigos mais importantes desse cluster, pode-se perceber que essa área de estudo visa mostrar os benefícios oriundo do alcance dos ODS, bem como da relação entre eles, que permitem ganhos ambientais, sociais e organizacionais. Então, a ser grupo foi dado o nome de: Interrelacionando os ODS.

Tabela 4 - Cluster 4 (Laranja).

\begin{tabular}{|c|c|c|c|}
\hline $\begin{array}{c}\mathrm{N}^{\circ} \text { de } \\
\text { Publicações }\end{array}$ & Autores com maior score & Artigo & Análise do Abstract \\
\hline \multirow{3}{*}{$\begin{array}{c}4^{\circ} \text { Cluster: } \\
37\end{array}$} & $\begin{array}{l}\text { Gerald G. Singh; Andrés M. } \\
\text { Cisneros-Montemayor; Wilf } \\
\text { Swartz; William Cheung; J. } \\
\text { Adam Guy; Tiff-Annie Kenny; } \\
\text { Chris J. McOwen; Rebecca Asch; } \\
\text { Jan Laurens Geffert; Colette C.C. } \\
\text { Wabnitz; Rashid Sumaila; } \\
\text { Quentin Hanich; Yoshitaka Ota } \\
\text { (score 14) }\end{array}$ & $\begin{array}{l}\text { A rapid assessment of } \\
\text { co-benefits and trade- } \\
\text { offs among Sustainable } \\
\text { Development Goals. }\end{array}$ & $\begin{array}{l}\text { Avalia as relações entre ODS, } \\
\text { destacando a importância dos } \\
\text { ecossistemas presentes nos oceanos. }\end{array}$ \\
\hline & $\begin{array}{l}\text { Francesco Rosati; Lourenço } \\
\text { Galvão Diniz Faria } \\
\text { (score 9) }\end{array}$ & $\begin{array}{l}\text { Business contribution to } \\
\text { the Sustainable } \\
\text { Development Agenda: } \\
\text { Organizational factors } \\
\text { related to early } \\
\text { adoption of SDG } \\
\text { reporting. }\end{array}$ & $\begin{array}{l}\text { Investiga a adoção de ODS nas } \\
\text { organizações e as características } \\
\text { estruturais destas. }\end{array}$ \\
\hline & $\begin{array}{l}\text { Mara Ntona; Elisa Morgera } \\
\text { (score 4) }\end{array}$ & $\begin{array}{l}\text { Connecting SDG } 14 \\
\text { with the other } \\
\text { Sustainable } \\
\text { Development Goals } \\
\text { through marine spatial } \\
\text { planning. }\end{array}$ & $\begin{array}{l}\text { Realiza interconexões entre os ODS, } \\
\text { destacando a conservação e o uso } \\
\text { sustentável dos oceanos para o } \\
\text { desenvolvimento sustentável. }\end{array}$ \\
\hline
\end{tabular}

Fonte: Autores, adaptado de CitNetExplorer (2020).

O cluster, representado pela cor amarela na Figura 3, contém 23 publicações. Diante do estudo feito nos abstracts das três principais publicações desse grupo, ficou claro que o tema pesquisado orbita sobre a sustentabilidade urbana, o que permitiu nomear o grupo como: Cidades Sustentáveis. 
Tabela 5 - Cluster 5 (Amarelo).

\begin{tabular}{|c|c|c|c|}
\hline $\begin{array}{c}\mathrm{N}^{\circ} \text { de } \\
\text { Publicaçōes }\end{array}$ & $\begin{array}{l}\text { Autores com } \\
\text { maior score }\end{array}$ & Artigo & Análise do Abstract \\
\hline \multirow{3}{*}{$\begin{array}{c}5^{\circ} \text { Cluster: } \\
23\end{array}$} & $\begin{array}{l}\text { Jacqueline M, } \\
\text { Klopp, Danielle L, } \\
\text { Petretta } \\
\text { (score 9) }\end{array}$ & $\begin{array}{l}\text { The urban sustainable development goal: } \\
\text { Indicators, complexity and the politics of } \\
\text { measuring cities. }\end{array}$ & $\begin{array}{l}\text { Trata do ODS voltado para o } \\
\text { desenvolvimento urbano } \\
\text { sustentável, enfatizando } \\
\text { algumas difieuldades } \\
\text { relacionadas aos seus } \\
\text { indieadores. }\end{array}$ \\
\hline & $\begin{array}{l}\text { Maria Kaika } \\
\text { (score 3) }\end{array}$ & $\begin{array}{l}\text { 'Don't call me resilient again!': the New } \\
\text { Urban Agenda as immunology ... or .... what } \\
\text { happens when communities refuse to be } \\
\text { vaccinated with 'smart cities 'and indicators. }\end{array}$ & $\begin{array}{l}\text { Busca um novo paradigma } \\
\text { urbano voltado para a } \\
\text { sustentabilidade. }\end{array}$ \\
\hline & $\begin{array}{l}\text { Roland } \\
\text { Zinkernagel; James } \\
\text { Evans; Lena Neij } \\
\text { (seore 3) }\end{array}$ & $\begin{array}{l}\text { Applying the SDGs to Cities: Business as } \\
\text { Usual or a New Dawn? }\end{array}$ & $\begin{array}{l}\text { Revisa a evolução dos } \\
\text { indieadores para monitorar o } \\
\text { desenvolvimento urbano } \\
\text { sustentável. }\end{array}$ \\
\hline
\end{tabular}

Fonte: Autores, adaptado de CitNetExplorer (2020).

A cor marrom na Tabela 6 representa o penúltimo cluster. Esse grupo possui 22 publicações, que versam sobre a aplicabilidade dos ODS, constituindo agendas de implementação destes. Assim, deu-se o nome a esse cluster de: Os ODS na prática.

Tabela 6 - Cluster 6 (Marrom).

\begin{tabular}{|c|c|c|c|}
\hline $\begin{array}{c}\mathrm{N}^{\circ} \text { de } \\
\text { Publicaçóes }\end{array}$ & $\begin{array}{c}\text { Autores com maior } \\
\text { score }\end{array}$ & Artigo & Análise do Abstract \\
\hline \multirow{3}{*}{$\begin{array}{l}6^{\circ} \text { Cluster: } \\
22\end{array}$} & $\begin{array}{l}\text { Jeffrey D. Sachs; } \\
\text { Guido Schmidt- } \\
\text { Traub; Mariana } \\
\text { Mazzucato; Dirk } \\
\text { Messner; Nebojsa } \\
\text { Nakicenovic; Johan } \\
\text { Rockström (score } 5 \text { ) }\end{array}$ & $\begin{array}{l}\text { Six Transformations to achieve the } \\
\text { Sustainable Development Goals. }\end{array}$ & $\begin{array}{l}\text { Traça uma agenda de ação, } \\
\text { fornecendo o conhecimento } \\
\text { necessário para projetar, } \\
\text { implementar e monitorar as } \\
\text { transfromaçōes nos ODS. }\end{array}$ \\
\hline & $\begin{array}{l}\text { Aysha Fleming; } \\
\text { Russell M. Wise; } \\
\text { Heidi Hansen; Linda } \\
\text { Sams (score 4) }\end{array}$ & $\begin{array}{l}\text { The sustainable development goals: A case } \\
\text { study. }\end{array}$ & $\begin{array}{l}\text { Apresenta um estudo prático } \\
\text { acerca da implementação dos } \\
\text { ODS numa empresa na } \\
\text { Austrália, mostrando suas } \\
\text { motivaçōes e barreiras. }\end{array}$ \\
\hline & $\begin{array}{l}\text { Theo Hacking } \\
\text { (score 3) }\end{array}$ & $\begin{array}{l}\text { The SDGs and the sustainability assessment } \\
\text { of private-sector projects: theoretical } \\
\text { conceptualisation and comparison with } \\
\text { current practice using the case study of the } \\
\text { Astan Development Bank. }\end{array}$ & $\begin{array}{l}\text { Explora o potencial de } \\
\text { desenvolver pesquisas } \\
\text { focadas nos ODS. }\end{array}$ \\
\hline
\end{tabular}

Fonte: Autores, adaptado de CitNetExplorer (2020).

O último cluster, representado pela cor rosa na Figura 3, é constituído por 21 publicações, sendo as três mais relevantes com score igual a 5. Diante da análise dos abstracts desses artigos de destaque no cluster, possibilitou o entendimento sobre a área de estudo desse grupo, cujo o enfoque é em estratégias políticas de governança voltadas à Bioeconomia. Assim, o cluster foi intitulado de: Bioeconomia a partir de Políticas Públicas. 
Tabela 7 - Cluster 7 (Rosa).

\begin{tabular}{|c|c|c|c|}
\hline $\begin{array}{c}\mathrm{N}^{\circ} \text { de } \\
\text { Publicações }\end{array}$ & $\begin{array}{c}\text { Autores com maior } \\
\text { score }\end{array}$ & Artigo & Análise do Abstract \\
\hline \multirow{3}{*}{$\begin{array}{l}7^{\circ} \text { Cluster: } \\
21\end{array}$} & $\begin{array}{l}\text { Thomas Dietz; Jan } \\
\text { Borner; Jan Janosch } \\
\text { Forster; Joachim Von } \\
\text { Braun (score } 5 \text { ) }\end{array}$ & $\begin{array}{l}\text { Governance of the Bioeconomy: A Global } \\
\text { Comparative Study of National Bioeconomy } \\
\text { Strategies. }\end{array}$ & $\begin{array}{l}\text { Diferencia quatro vias de } \\
\text { desenvolvimento } \\
\text { bioeconômico, a partir de } \\
\text { mecanismos eficazes de } \\
\text { governança. }\end{array}$ \\
\hline & $\begin{array}{l}\text { Walther Zeug; } \\
\text { Alberto Bezama; Urs } \\
\text { Moesenfechtel; Anne } \\
\text { Jahkel; Daniela Thrän } \\
\text { (score 5) }\end{array}$ & $\begin{array}{l}\text { Stakeholders' Interests and Perceptions of } \\
\text { Bioeconomy Monitoring Using a } \\
\text { Sustainable Development Goal Framework. }\end{array}$ & $\begin{array}{l}\text { Avaliar a relevancia dos ODS } \\
\text { para a bioeconomia. }\end{array}$ \\
\hline & $\begin{array}{l}\text { Maria Bastida; } \\
\text { Alberto Vaquero } \\
\text { Gareia; Maite } \\
\text { Cancelo Márquez; } \\
\text { Ana Olveira Blanco } \\
\text { (score 5) }\end{array}$ & $\begin{array}{l}\text { Fostering the Sustainable Development } \\
\text { Goals from an Ecosystem Conducive to the } \\
\text { SE: The Galician's Case. }\end{array}$ & $\begin{array}{l}\text { Expliea a estratégia de } \\
\text { implementação da Economia } \\
\text { Social em Galiza, a partir de } \\
\text { politicas públicas. }\end{array}$ \\
\hline
\end{tabular}

Fonte: Autores, adaptado de CitNetExplorer (2020).

\subsection{Das publicações que não formaram clusters}

Como foi exposto a priori, a análise de Clustering no Core Publications, formou 7 clusters, sendo que 665 publicações não se enquadraram em nenhum cluster. Visando investigar a ocorrência desse dado, foi feito o h-index dessas publicações no próprio software, que retornou 3 publicações para análise, como demonstrado na Figura 4.

Figura 4 - H-index das publicações que não formaram clusters.

\begin{tabular}{|c|c|c|c|}
\hline Luthers & Soute & Fee & Ctrowe v \\
\hline 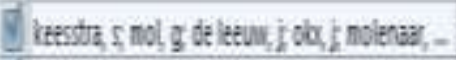 & 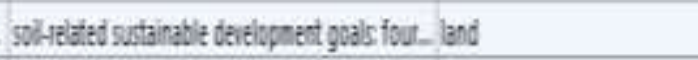 & 2018 & 5 \\
\hline 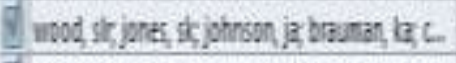 & 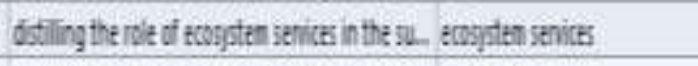 & 218 & 5 \\
\hline Itees se, foster, nt langnead, o, pitman r, john. & 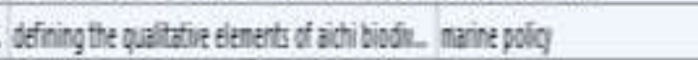 & 218 & 3 \\
\hline
\end{tabular}

Fonte: Autores, adaptado de CitNetExplorer (2020).

Visando elucidar o não enquadramento dessas publicações nos clusters gerados, foi realizada a análise dos abstracts das três publicações selecionadas no h-index, como evidencia a Tabela 8. O resultado obtido confirma o dado fornecido pelo software, haja vista que os temas abordados nessas publicações não estão relacionados diretamente com o objeto de estudo Gestão Municipal voltada para os Objetivos de Desenvolvimento Sustentável à luz de Indicadores de Sustentabilidade - mas sim, a temas emergentes relacionados aos ODS, como o LDN (Neutralização de Degradação da Terra), serviços ecossistêmicos e Meta 11 de Aichi. 
Tabela 8 - Publicações que não formaram clusters.

\begin{tabular}{|c|c|c|}
\hline Score & Artigos & Análise do Abstract \\
\hline 5 & $\begin{array}{l}\text { Soil-Related Sustainable Development Goals: Four } \\
\text { Concepts to Make Land Degradation Neutrality and } \\
\text { Restoration Work. } \\
\text { Saskia Keesstra; Gerben Mol; Jan De Leeuw; Joop Okx; } \\
\text { Co Molenaar; Margot De Cleen; Saskia Visser }\end{array}$ & 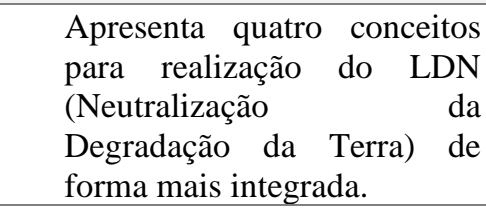 \\
\hline 5 & $\begin{array}{l}\text { Distilling the role of ecosystem services in the } \\
\text { Sustainable Development Goals. } \\
\text { Sylvia L. R. Wood; Sarah K. Jones; Justin A. Johnson; } \\
\text { Kate A. Brauman; Rebecca Chaplin-Kramer; Alexandre } \\
\text { Fremier; Evan Girvetz; Line J. Gordon; Carrie V. Kappel; } \\
\text { Lisa Mandle; Mark Mulligan; Patrick O’Farrell; William } \\
\text { K. Smith; Louise Willemen; Wei Zhang; Fabrice A. } \\
\text { DeClerck }\end{array}$ & $\begin{array}{l}\text { Apresenta uma pesquisa sobre as } \\
\text { contribuições de } 16 \text { serviços } \\
\text { ecossistêmicos para o alcance os } \\
\text { ODS. }\end{array}$ \\
\hline 3 & $\begin{array}{l}\text { Defining the qualitative elements of Aichi Biodiversity } \\
\text { Target } 11 \text { with regard to the marine and coastal } \\
\text { environment in order to strengthen global efforts for } \\
\text { marine biodiversity conservation outlined in the United } \\
\text { Nations Sustainable Development Goal 14. } \\
\text { Siân E. Rees; Nicola L. Foster; Olivia Langmead; Simon } \\
\text { Pittman; David E. Johnson }\end{array}$ & $\begin{array}{l}\text { Apresenta o conhecimento atual } \\
\text { e a implementação dos elementos } \\
\text { qualitativos da Meta } 11 \text { de Aichi } \\
\text { e destaca lacunas no } \\
\text { conhecimento. }\end{array}$ \\
\hline
\end{tabular}

Fonte: Autores, adaptado de CitNetExplorer (2020).

\subsection{Dos artigos do $h$-index}

O h-index gerado com as informações do próprio CitNetExplorer, viabilizou analisar os 7 principais artigos da base de dados escolhida, tais artigos, dos anos de 2017 a 2019, apresentaram scores variando de 9 a 15, como é evidenciado na Figura 5.

Figura 5 - H-index.

\begin{tabular}{|c|c|c|c|c|c|}
\hline All putlictions 954) & Selected publications: & Marted publications & & & \\
\hline Authors & & Trle & Source & Iear & Ct sener \\
\hline spangenberg. in & het ar or conpreher & nsive progrest? a uitical assesment of the sdgs & gustairable dealogment & 2017 & 15 \\
\hline 7 gingh $9 g$ disneras & - 1 agid assessment 8 & of co-benefilts and tade-sfft anong soutainable develagnent guals & raine polig & 2018 & 14 \\
\hline 7 holden, 5 linnerud. & - the inperatives of su. & utairable dertiognent & gustainable derelopment & 2017 & II \\
\hline dan-sarschage, inj, & L is the sustainable de & eilogment guals fidgl inder an adequate fianework to neasure the proquess o. & rustairable deislopnent & 2018 & 11 \\
\hline 3 janouskase r hak:- & - global udgr asserim. & ents helping or confusing indicatur? & sutarability & 2012 & II \\
\hline J Woph, in petuetta d & d. the utban surtainab & le dejelop-nert goat: indicator, campleaty and the polities of neasuring oties & ctost & 2017 & 9 \\
\hline 7 rosah $f$ fara, ligd & business contributio & 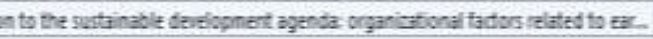 & carporate sucial respons. & 2019 & 9 \\
\hline
\end{tabular}

Fonte: Autores, adaptado de CitNetExplorer (2020).

Visando construir um entendimento acerca do conceito, ainda pouco definido na literatura, sobre Desenvolvimento Sustentável, tema central do estudo proposto, foi realizada uma análise dos 7 artigos revelados no h-index. Assim, a partir da leitura desses artigos, foi construído o Quadro 1 que apresenta os conceitos e as características designadas ao Desenvolvimento Sustentável nos artigos revisados. 
Quadro 1 - Conceitos e características do desenvolvimento sustentável a partir artigos do h-index.

Conceitos e características do desenvolvimento sustentável

Artigo 1: Hot air or comprehensive progress? (Spagenberg, 2017)

"Satisfazer as necessidades humanas, em particular as dos mais pobres, e aceitar limitações na criação de pressões ambientais.” (p. 312)

"Endossar o livre comércio sem quaisquer ressalvas, mercados livres sem mencionar seus efeitos ecológicos e sociais e crescimento econômico sem limitações ignora os dois princípios básicos do desenvolvimento sustentável.” (p. 315)

“... mudar o papel atribuído aos negócios no desenvolvimento sustentável, de fornecer meios a objetivos politicamente determinados para ser um agente desempenhando um papel proeminente nos objetivos e estratégias." (p. 316)

"O papel que atribui aos governos está em preparar o caminho para que as empresas entreguem o desenvolvimento sustentável.” (p. 316)

"Apoiar a educação em todos os níveis foi reconhecido como uma condição para o desenvolvimento sustentável” (p.317)

“... a virada para o desenvolvimento sustentável não acontecerá por si só, nem será provocada apenas pelos mercados: é necessária uma ação política decisiva.” (p.319)

"Quem quer um desenvolvimento sustentável substancial não pode deixar de optar por estabelecer regras e padrões juridicamente vinculativos para todos os agentes relevantes (incluindo negócios), por intervenção nos processos de mercado, por regulamentação em vez de desregulamentação e por um papel forte de cidadãos e tudo grupos principais." (p.319)

“... instituições devem passar por uma verificação de sustentabilidade...” (p. 319)

“... desempenho empresarial que contribua para o desenvolvimento sustentável...” (p. 319)

\section{Artigo 2: A rapid assessment of co-benefits and trade-offs among Sustainable Development Goals} (Singh et al., 2018)

"Alcançar o desenvolvimento sustentável enfrenta muitos desafios ecológicos e sociais, como gestão de recursos, escassez de recursos, contaminação ambiental e persistência nesse trabalho.” (p. 223)

“... conservar e usar de forma sustentável os oceanos, mares e recursos marinhos para o desenvolvimento sustentável.” (p. 224)

“... metas de desenvolvimento sustentável exigem que a sustentabilidade do oceano seja alcançada." (p.229)

“... a natureza interrelacionada do desenvolvimento sustentável.” (p. 230)

Artigo 3: The imperatives of sustainable development (Holden, Linnerud \& Banister, 2017)

"O desenvolvimento sustentável constitui um conjunto de restrições ao comportamento humano, incluindo restrições à atividade econômica.” (p. 213)

"Qualquer noção de desenvolvimento sustentável deve reconhecer os limites ambientais..." (p. 213)

"O desenvolvimento sustentável é um sistema de valores normativos, em pé de igualdade com os direitos humanos, a democracia e a liberdade (e está intimamente interligado com todos esses sistemas)." (p. 215)

“... o desenvolvimento sustentável é essencialmente um forte pronunciamento ético ou moral sobre o que deve ser feito." (p. 215)

"O conceito de desenvolvimento sustentável se apoia em três imperativos morais: satisfazer as necessidades humanas, garantir a equidade social e respeitar os limites ambientais." (p. 215)

“... política que leva ao desenvolvimento sustentável é aquela que atinge uma meta socialmente desejável...” (p. 215)

"O crescimento econômico não é uma das principais dimensões do desenvolvimento sustentável. É verdade que o crescimento econômico pode contribuir para um desenvolvimento mais sustentável melhorando o bemestar social, satisfazendo as necessidades humanas e tirando as pessoas da pobreza, mas o crescimento econômico também pode reduzir a igualdade social ao contribuir para a desigualdade de renda e riqueza." (p. 216) 
“... a igualdade social, incluindo a participação democrática, é um tema-chave do desenvolvimento sustentável” (p. 216)

“... tem faltado consenso sobre como medir a sustentabilidade" (p. 220)

“O desenvolvimento sustentável global, nacional e local deve abordar temas-chave globais, nacionais e locais, respectivamente.” (p .222)

"Não pode haver desenvolvimento sustentável sem paz e não pode haver paz sem desenvolvimento sustentável" (p. 222)

\section{Artigo 4: Is the Sustainable Development Goals (SDG) index an adequate framework to measure the progress of the 2030 Agenda? \\ (Diaz-Sarachaga, Jato-Espino \& Castro-Fresno, 2018)}

"Os indicadores de sustentabilidade surgiram para satisfazer a necessidade de medir o progresso do desenvolvimento sustentável e facilitar os processos de tomada de decisão sob a consideração de suas três dimensões.” (p. 663)

"O desenvolvimento sustentável é um conceito pouco claro que pode levar a mal-entendidos devido às suas múltiplas e variadas definições. No entanto, há um consenso geral sobre sua complexidade, incerteza e caráter multidimensional, o que requer uma análise aprofundada de diferentes magnitudes (tempo, espaço, função), múltiplos atores (econômicos, sociais) e diversas interrelações sistêmicas.” (p. 663)

“O termo desenvolvimento sustentável foi cunhado na tentativa de vincular o meio ambiente ao desenvolvimento, sendo definido como o desenvolvimento que atende às necessidades da geração atual sem comprometer a capacidade das gerações futuras de atender às suas próprias necessidades.” (p. 663)

"A natureza multidisciplinar do desenvolvimento sustentável e as dificuldades em reconhecer seu significado dificultam muito seu monitoramento por meio de indicadores únicos.” (p. 664)

"Os avanços subsequentes na compreensão do conceito multidimensional de desenvolvimento sustentável ajudaram a determinar uma ampla gama de interrelações entre o meio ambiente, a sociedade e a economia." (p. 669)

\section{Artigo 5: Global SDGs assessments: Helping or confusing indicators?}

(Janoušková, Hák \& Moldan, 2018)

"Os indicadores desempenham um papel fundamental em tornar o conceito de desenvolvimento sustentável (DS) atraente para um amplo espectro de partes interessadas em potencial, bem como na avaliação do progresso.” (p. 1)

"O desenvolvimento sustentável, entendido como um processo social de exploração, aprendizagem e moldagem do futuro, envolve necessariamente comunicação.” (p. 2)

“... desenvolvimento sustentável é um processo de múltiplas partes interessadas.” (p. 3)

“A forma pragmática de definição de DS relaciona-se com a sua “medição” e inclui indicadores...” (p. 3)

\section{Artigo 6: The urban sustainable development goal: Indicators, complexity and the politics of measuring} cities (Klopp \& Petretta, 2017)

“... as cidades também estão sendo conceituadas menos como problemas e mais como impulsionadores do desenvolvimento sustentável e mudança ambiental global.” (p. 92)

“... no mundo urbano, as cidades podem ser caminhos para o desenvolvimento sustentável.” (p. 93)

\section{Artigo 7: Business contribution to the Sustainable Development Agenda: Organizational factors related to early adoption of SDG reporting (Rosati \& Faria, 2019)}

"Um grande desafio na implementação da Agenda de Desenvolvimento Sustentável é mudar as atitudes das empresas em relação a novas práticas, tecnologias e modelos de negócios de sustentabilidade.” (p. 588)

“... a discussão dominante sobre o desenvolvimento sustentável é bastante recente.” (p. 591)

“Os indivíduos mais jovens são expostos mais cedo ao desenvolvimento sustentável e possuem mais conhecimento sobre ele.” (p. 591) 
É unânime a dificuldade em encontrar uma definição de Desenvolvimento Sustentável que abranja toda sua complexidade, fato reforçado com a leitura dos 7 artigos. Percebe-se que cada estudo se inclina para um dos três aspectos principais da sustentabilidade - econômico, social e ambiental.

No primeiro artigo, o conceito de Desenvolvimento Sustentável enfoca a questão social e no objetivo de satisfazer as necessidades humanas. Intervenção nos processos de mercados, investimentos em educação e uma ação política decisiva, são aspectos importantes para o alcance do Desenvolvimento Sustentável segundo esse estudo.

O segundo artigo, traz as questões ambientais como centro das discussões para o Desenvolvimento Sustentável, aqui, a gestão de recursos e a conservação é pauta principal. Já, e acordo com o terceiro artigo, o Desenvolvimento Sustentável é marcado por restrições ao comportamento humano destrutivo e à atividade econômica desmedida, estando intrinsecamente relacionado aos direitos humanos, democracia, liberdade, ética e moralidade.

$\mathrm{O}$ quarto artigo aborda os indicadores de sustentabilidade como fortes aliados na tentativa de mensurar esse Desenvolvimento Sustentável, marcado por múltiplas definições e por sua natureza multidisciplinar. O quinto artigo reitera a essencialidade desses indicadores e envolve a comunicação como aspectos relevante no processo de Desenvolvimento Sustentável, o qual envolve várias partes interessadas.

O sexto artigo discute o caráter impulsionador das cidades no contexto de Desenvolvimento Sustentável, como um caminho real para o seu alcance. O Sétimo e último artigo dessa lista alerta para que haja uma mudança no meio empresarial em prol da sustentabilidade, destacando que o Desenvolvimento Sustentável é uma discussão ainda embrionária e que os mais jovens estão expostos mais cedo a ela.

\section{Considerações Finais}

Esta pesquisa se debruçou na análise da produção científica recente sobre os temas - Objetivos de Desenvolvimento Sustentável, Indicadores de Sustentabilidade e Gestão Municipal Sustentável - a fim de compreendê-los e identificar os principais contextos que orbitam sobre os temas em estudo.

Como exposto, a definição de desenvolvimento sustentável é complexa e controversa, demandando uma mudança no modo de avaliar a realidade, nos meios de convivência, produção, consumo e, consequentemente, na gestão (Marques et al., 2020).

Diante dessa problemática, o presente estudo permitiu evidenciar que o entendimento do desenvolvimento sustentável, apontado na literatura analisada, converge para um sistema dotado de variações e complexidades e se caracteriza por várias dimensões, mas que, embora apresentem especificidades, elas são interdependentes, não sendo possível isolá-las.

Os textos explorados nesse estudo revelaram não só temáticas associadas aos Objetivos de Desenvolvimento Sustentável, aos Indicadores de Sustentabilidade e à Gestão Pública Sustentável, como também, enfoques em educação, bioeconomia, cidades sustentáveis e estudos sobre a prática dos ODS, corroborando, assim, com a multidisciplinariedade do tema.

Ao passo que concretiza a relevância e a grandeza do tema abordado nessa pesquisa, os resultados marcados pela heterogeneidade foram também uma limitação nesse estudo, pois demandou grande esforço para agrupar os trabalhos e classificá-los nos clusters, tendo em vista as múltiplas perspectivas abordadas.

Por fim, destaca-se alguns assuntos transversais, que não dialogaram diretamente com o objeto de estudo proposto, mas que foram identificados durante a coleta de dados, viabilizando, dessa forma, novos estudos, com outras perspectivas e diferentes abordagens, mas com o mesmo tema central, relevante e contemporâneo aqui abordado: Desenvolvimento Sustentável. 


\section{Referências}

Carvalho, J. R. M., Curi, W. F., Carvalho, E. K. M. A., \& Curi, R. C. (2011). Proposta e validação de indicadores hidroambientais para bacias hidrográficas: estudo de caso na sub-bacia do alto curso do rio Paraíba, PB. Sociedade \& Natureza, 23(2), 295-310. http://www.seer.ufu.br/index.php/sociedadenatureza/article/view/12517

Carvalho, P. G. M., \& Barcellos, F. C. (2010). Mensurando a sustentabilidade. Economia do meio ambiente: teoria e prática. Campus-Elsevier.

CitNetExplorer (2020). (Versão 1.0.0). Leiden University.

Diaz-Sarachaga, J. M., Jato-Espino, D., \& Castro-Fresno, D. (2018). Is the sustainable development goals (SDG) index an adequate framework to measure the progress of the 2030 agenda? Sustainable Development, 26(6), 663-671.

Fórum AG21 (2000). Agenda 21 Local do Município de Florianópolis: meio ambiente quem faz é a gente. Prefeitura Municipal de Florianópolis.

Gallopin, G. C. (1996). Environmental and sustainability indicators and the concept of situational indicators. A systems approach. Environmental modeling \& assessment, 1(3), 101-117. https://link.springer.com/article/10.1007/BF01874899

Hammond, A. (1995). Environmental indicators: a systematic approach to measuring and reporting on environmental policy performance in the context of sustainable development (Vol. 36). World Resources Institute.

Holden, E., Linnerud, K., \& Banister, D. (2017). The imperatives of sustainable development. Sustainable Development, 25(3), $213-226$.

Jacobi, P. (1999). Meio ambiente e sustentabilidade. In Fundação Prefeito Faria Lima, O município no século XXI: cenários e perspectivas (pp. 175-184). CEPAM.

Janoušková, S., Hák, T., \& Moldan, B. (2018). Global SDGs assessments: helping or confusing indicators? Sustainability, 10(5), 1540.

Kemerich, P. D. C., Ritter, L. G., \& Borba, W. F. (2014). Indicadores de sustentabilidade ambiental: métodos e aplicações. Revista Monografias Ambientais, 13(4), 3718-3722. https://periodicos.ufsm.br/remoa/article/view/14411/0. 10.5902/2236130814411

Kitchenham, B., \& Charters, S. (2007). Guidelines for performing systematic literature reviews in software engineering. University of Durham.

Klopp, J. M., \& Petretta, D. L. (2017). The urban sustainable development goal: indicators, complexity and the politics of measuring cities. $63,92-97$.

Lopes, S., Costa, M. T., Fernández-Llimós, F., Amante, M. J., \& Lopes, P. F. (2012, outubro). A Bibliometria e a Avaliação da Produção Científica: indicadores e ferramentas. Anais do Congresso Nacional de Bibliotecários, Arquivistas e Documentalistas, https://www.bad.pt/publicacoes/index.php/congressosbad/article/view/429

Marques, J. F. S., Santos, Â. V., \& Aragão, J. M. C. (2020). Planejamento e sustentabilidade em instituições de ensino superior à luz dos objetivos do desenvolvimento sustentável. REUNIR Revista de Administração, Contabilidade e Sustentabilidade, 10(1), 14-29. https://reunir.revistas.ufcg.edu.br/index.php/uacc/article/view/1052

Menezes, H. Z., \& Minillo, X. K. (2017). Pesquisa e extensão como contribuição da universidade na implementação dos objetivos de desenvolvimento sustentável (ODS) no Brasil. Meridiano 47, 18, 1-16. https://biblat.unam.mx/pt/revista/meridiano-47/articulo/pesquisa-e-extensao-como-contribuicao-dauniversidade-na-implementacao-dos-objetivos-de-desenvolvimento-sustentavel-ods-no-brasil

Moura, S. (1998). A gestão do desenvolvimento local estratégias e possibilidades de financiamento. Organizações \& Sociedade, 5(12), 37-57. https://www.scielo.br/scielo.php?script=sci_arttext\&pid=S1984-92301998000200002. doi: 10.1590/S1984-92301998000200002

Organização das Nações Unidas $(n$. d.). Sobre o nosso trabalho para alcançar os objetivos de desenvolvimento sustentável no Brasil [Blog]. https://brasil.un.org/pt-br/sdgs

Polaz, C. N. M., \& Teixeira, B. A. N. (2009). Indicadores de sustentabilidade como ferramenta para a gestão municipal de resíduos sólidos: um estudo para São Carlos (SP). Engenharia Sanitária e Ambiental, 4(3), 411-420. https://www.scielo.br/pdf/esa/v14n3/v14n3a15.pdf. 10.1590/S1413-41522009000300015

Réus, I., \& Andion, C. (2018). Gestão municipal e desenvolvimento sustentável: panorama dos indicadores de sustentabilidade nos municípios catarinen ses. Desenvolvimento em Questão, 16(45), 97-117. Recuperado de https://www.revistas.unijui.edu.br/index.php/desenvolvimentoemquestao/article/view/6177. $10.21527 / 2237-6453.2018 .45 .97-117$

Rosati, F., \& Faria, L. G. (2019). Business contribution to the sustainable development agenda: organizational factors related to early adoption of SDG reporting. Corporate Social Responsibility and Environmental Management, 26(3), 588-597.

Schenini, P. C., \& Nascimento, D. T. (2002). Gestão pública sustentável. Revista de Ciências da Administração, 4 (8), 1-18. https://periodicos.ufsc.br/index.php/adm/article/view/1920. doi: 10.5007/\%25x

Singh, G. G., Cisneros-Montemayor, A. M., Swartz, W., Cheung, W., Guy, J. A., Kenny, T. A., \& Sumaila, R. (2018). A rapid assessment of co-benefits and trade-offs among sustainable development goals. Marine Policy, 93, 223-231.

Spangenberg, J. H. (2017). Hot air or comprehensive progress? A critical assessment of the SDGs. Sustainable Development, 25(4), 311-321.

Van Bellen, H. M. (2004). Desenvolvimento sustentável: uma descrição das principais ferramentas de avaliação. Ambiente \& Sociedade, 7(1), 67-87. https://www.scielo.br/scielo.php?pid=S1414-753X2004000100005\&script=sci_abstract\&tlng=pt. doi: 10.1590/S1414-753X2004000100005

Van Bellen, H. M. (2006). Indicadores de sustentabilidade: uma análise comparativa. (2a ed.). Editora FGV.

Web of Science (2020). Recuperado de https://www.webofknowledge.com 